\title{
Optimal Skills Desirable to Identify Multiple Alloantibodies in a Patient
} Manish Raturi* and Savina Prasad

Department of Immunohematology and Blood Transfusion, Kasturba Medical College, Manipal Academy of Higher Education, Manipal, Karnataka, India

\begin{abstract}
Background: Multiple alloantibodies in patients' sera present with widely variable reaction strengths and matching patterns. Therefore, from an immuno-haematologists' point of view, alloantibodies once detected in antenatal or pre-transfusion setting, must be confirmed for their specificity and clinical significance.

Case report: We report herein the case of a 57-year old female patient admitted in our hospital with chief complaints of breathlessness and mild grade fever. She was a known case of Type II diabetes mellitus with endstage renal disease. Her requisition for one pint of packed red blood cell (PRBC) was received at our department for pre-transfusion compatibility test and subsequent issue of blood. During immunohematology work-up, her blood type was found to be B Rh D Positive. However, her sera showed incompatibility with random B Rh D positive PRBC units. Her indirect coombs test was positive. The reactivity pattern in an eleven cell identification panel (Biorad, Switzerland) showed variable pattern and suggested of antibody/s against $E$ and $c$ antigen specificity. We subsequently issued one $B$ Rh D positive ( $E$ and $c$ antigen negative) anti-human globulin cross-matched compatible PRBC to the patient. We also issued an antibody card for her future reference.

Conclusion: Transfusion of phenotypically matched PRBC for the implicated $E$ and $c$ compared to that phenotypically matched for the standard ABO-D System could help save patient from adverse transfusion event/s. Knowledge of multiple alloantibodies can assist in selecting appropriate transfusion strategy for the patient/s. In addition, requisite skill and precision is always desirable when dealing with multiple allo-antibodies because they can directly influence patient's clinical outcome.
\end{abstract}

Keywords: Multiple alloantibodies; Immunohematology; Laboratory; Guidelines; Blood Transfusion

\section{Introduction}

Erythrocyte antigens and alloantibodies differ significantly among varied human populations and ethnic groups [1]. Further allo-immunization after exposure to foreign antigens depends on genetic and or acquired patient-related factors (such as age, gender, antenatal settings, transfusion and or transplantation), total dose and the immunogenicity of the particular antigen [2]. In some instances, reasons are simply unknown. Their detection is often determined by the sensitivity of the testing methodology used. Clinically significant antibodies are capable of causing mild or severe adverse events following transfusion including haemolytic disease of the foetus and newborn [3]. Therefore, from an immuno-haematologist's point of view, alloantibodies once detected in antenatal or pre-transfusion setting, must be confirmed for their specificity and clinical significance. This is also vital since knowledge of such alloantibodies can assist in selecting appropriate transfusion strategy for the patient/s. The recipients should receive a packed red blood cell (PRBC) unit that has been tested to lack the corresponding antigen/s. In addition, determining the specificity of a single alloantibody that yields clear positive and negative reactions, generally imposes less difficulties. However, when it comes to multiple alloantibodies, interpretation of test results, invariably requires much more proficient skill and vigilance on the part of laboratory personnel involved. With this background we report herein a case of a patient who had multiple alloantibodies and required appropriate transfusion management.

\section{Case Report}

A 57-y old female patient Mrs. X was admitted at our hospital with chief complaints of breathlessness with mild grade fever. She was a known case of Type II diabetes mellitus with end-stage renal disease. Her requisition for one pint of $\mathrm{PRBC}$ was received at our department for pre-transfusion compatibility test and subsequent issue of blood. During immunohematology work-up, her blood type was found to be $\mathrm{B}$ $\mathrm{Rh} D$ Positive. However, her sera showed incompatibility with random B Rh D positive PRBC units. Her indirect coombs test was positive. Eleven-cell identification panel (Biorad, Switzerland) showed grade 4+ positive reaction with panel cells 3 and 5 and grade $2+$ positive reaction with panel cells $6,7,8,9,10$ and 11 respectively. This reactivity pattern was suggestive of antibodies against $\mathrm{E}$ and $\mathrm{c}$ antigen specificity (Figure 1). Patient's red cell phenotyping for $E$ and $c$ antigens was negative. This confirmed the presence of anti-c and anti-E allo-antibodies in her sera. On performing serial dilutions using her sera, the antibody titres obtained were 4 and 2 corresponding to both antigens (E and C) respectively.

\section{Therapeutic Support}

Multiple allo-antibodies in her sera warranted appropriate transfusion management after optimal detection and identification by the laboratory technician. We subsequently issued one $\mathrm{B} R \mathrm{Rh}$ positive ( $\mathrm{E}$ and $\mathrm{c}$ antigen negative) anti-human globulin cross-matched compatible PRBC to the patient. We also issued an antibody card for her future reference. Patient was followed-up during her hospital stay. At the time of discharge her haemoglobin was $10.2 \mathrm{~g} \%$ and her general

*Corresponding author: Manish Raturi, Department of Immunohematology nd Blood Transfusion, Kasturba Medical College, Manipal Academy of Higher Education, Manipal, Karnataka, India, Tel: +91-9897212766; E-mail: manishnsr@ gmail.com

Received November 06, 2018; Accepted November 13, 2018; Published November 19, 2018

Citation: Raturi M, Prasad S (2018) Optimal Skills Desirable to Identify Multiple Alloantibodies in a Patient. J Blood Lymph S1: 005. doi: 10.4172/2165-7831.S1-005

Copyright: (C) 2018 Raturi M, et al. This is an open-access article distributed under the terms of the Creative Commons Attribution License, which permits unrestricted use, distribution, and reproduction in any medium, provided the original author and source are credited. 


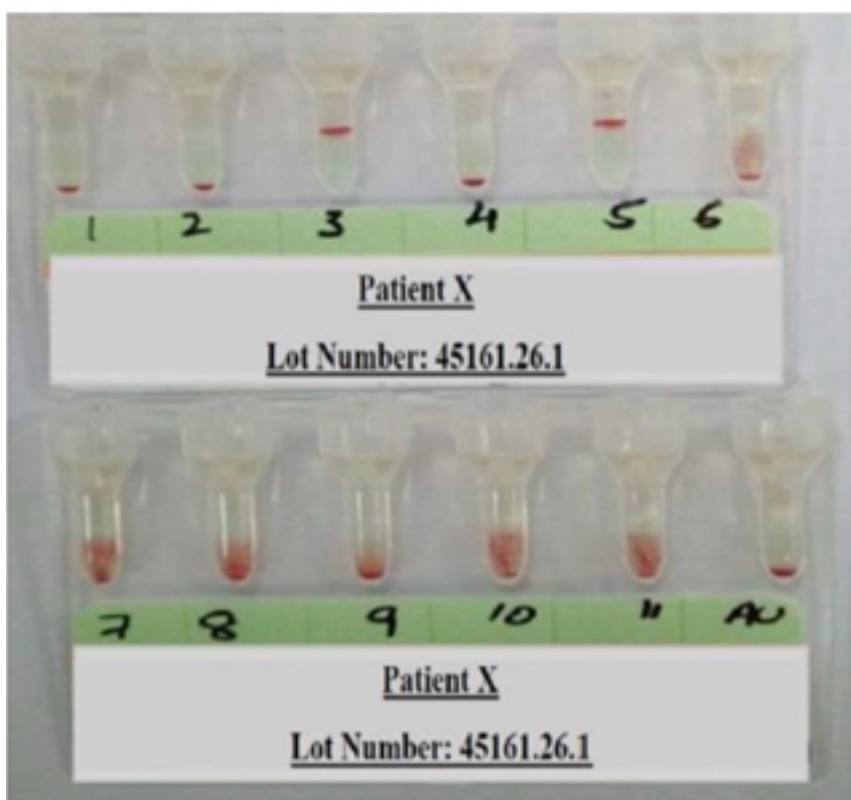

Figure 1: Multiple allo-antibodies showing pattern and dosage in the patient.

condition was significantly improved. British committee for standards in hematology (BCSH) invariably state that multiple alloantibodies can impose a vital challenge for the laboratory personnel due to their widely variable reaction strengths and matching patterns [4]. Transfusion of phenotypically matched $\mathrm{PRBC}$ for the implicated $\mathrm{E}$ and $\mathrm{c}$ compared to that phenotypically matched for the standard ABO-D System could help save patient from adverse transfusion event/s. Knowledge of multiple alloantibodies can assist in selecting appropriate transfusion strategy for the patient/s. In addition, requisite skill and precision is always desirable when dealing with multiple allo-antibodies because they can directly influence patient's clinical outcome.

\section{Compliance with Ethical Standards}

Research involving human participants and/or animals: Human participant.

Informed consent: As per the hospital transfusion protocol informed consent is obtained from all the patients prior to receiving blood transfusion itself.

Ethical approval: All procedures performed in studies involving human participants were in accordance with the ethical standards of the institutional ethics committee and with the 1964 Helsinki declaration and its later amendments or comparable ethical standards.

Authorship contributions: Savina Prasad contributed to the Immunohematology work-up and Dr. Manish contributed towards drafting the paper and its critical evaluation.

\section{References}

1. Cartron JP, Collin Y (2001) Structural and Functional Diversity of blood group antigens. Transfus Clin Biol 8: 163-199.

2. Siegel DL (1998) The human immune response to red blood cell antigens a revealed by repertoire cloning. Immunol Res 17: 3239-3251.

3. Poole J, Daniels G (2007) Blood Group Antibodies and Their Significance in Transfusion Medicine. Transfus Med Rev 21: 58-71.

4. Milkins C, Berryman J, Cantwell C, Elliott C, Haggas R, et al. (2013) Guidelines for pre-transfusion compatibility procedures in blood transfusion laboratories. British Committee for Standards in Haematology. Transfus Med 23: 3-35. 